\title{
Cognitive Mappers to Creatures of Habit: Differential Engagement of Place and Response Learning Mechanisms Predicts Human Navigational Behavior
}

\author{
Steven A. Marchette, ${ }^{\star}$ Arnold Bakker, ${ }^{\star}$ and Amy L. Shelton \\ Department of Psychological \& Brain Sciences, The Johns Hopkins University, Baltimore, Maryland 21218
}

Learning to navigate plays an integral role in the survival of humans and other animals. Research on human navigation has largely focused on how we deliberately map out our world. However, many of us also have experiences of navigating on "autopilot" or out of habit. Animal models have identified this cognitive mapping versus habit learning as two dissociable systems for learning a space-a hippocampal place-learning system and a striatal response-learning system. Here, we use this dichotomy in humans to understand variability in navigational style by demonstrating that brain activation during spatial encoding can predict where a person's behavior falls on a continuum from a more flexible cognitive map-like strategy to a more rigid creature-of-habit approach. These findings bridge the wealth of knowledge gained from animal models and the study of human behavior, opening the door to a more comprehensive understanding of variability in human spatial learning and navigation.

\section{Introduction}

A striking observation in human navigation is that individuals vary greatly in how they get around in the world. Anecdotally, people report everything from thinking in mental maps to establishing familiar routes to piecemeal wandering among landmarks. Research into navigational styles has developed broad categories for self-reported strategic preferences (Lawton, 1994; Pazzaglia and De Beni, 2001), but little is known about why these differences emerge at all-why should two people approach the same navigational problem in dramatically different ways? We offer a new approach to this question by asking how underlying neural mechanisms may give rise to different strategies.

Since the mid-20th century, we have known that rats exhibit two types of spatial learning (Tolman, 1948; Restle, 1957; Morris, 1981; Packard et al., 1989). For example, in the simple T-maze preparation (Fig. 1, top), the animal repeatedly searches for a goal in a maze with stable start and goal locations, such that the path to the goal is the same each time. At test, the T-maze is reversed relative to the environmental cues. When the animal navigates to the correct location relative to the environment, he shows place learning. Alternatively, when the animal navigates by taking the repeated path (i.e., turns left), he shows response learning. Place

\footnotetext{
Received July 14, 2011; revised Aug. 16, 2011; accepted Aug. 30, 2011.

Author contributions: S.A.M., A.B., and A.L.S. designed research; S.A.M. and A.B. performed research; S.A.M., A.B., and A.L.S. analyzed data; S.A.M., A.B., and A.L.S. wrote the paper.

This work was supported by National Science Foundation Cognitive Neuroscience Grant 0920221 to A.L.S. We thank A. Clements-Stephens and M. Yassa for critical discussion and comments; and J. Lachewitz and S. Clark for data collection.

*S.A.M. and A.B. contributed equally to this work.

Correspondence should be addressed to Amy L. Shelton, Department of Psychological \& Brain Sciences, The Johns Hopkins University, 3400 N. Charles Street, Baltimore, MD 21218. E-mail: ashelton@jhu.edu.

DOI:10.1523/JNEUROSCI.3634-11.2011

Copyright $\odot 2011$ the authors $\quad 0270-6474 / 11 / 3115264-05 \$ 15.00 / 0$
}

and response learning in rodents differ with respect to many behavioral characteristics (Tolman et al., 1946; Ritchie et al., 1950; Restle, 1957; O’Keefe and Nadel, 1978; Sage and Knowlton, 2000; Yin and Knowlton, 2006; Korol and Gold, 2007) and show a double dissociation in the brain: deactivation of the hippocampus reduces place performance with intact response performance, whereas deactivation of the caudate nucleus reduces response performance with intact place performance (Packard and McGaugh, 1996).

Support for the existence of place and response learning in humans comes from studies showing the engagement of hippocampus and caudate during various navigational tasks (Hartley et al., 2003; Iaria et al., 2003; Voermans et al., 2004; Iglói et al., 2010). Here, we take the innovative step of asking whether preferential engagement of these systems might be useful in characterizing and accounting for individual variability in navigational styles. In particular, we suggest that different explicit strategies such as mental maps versus familiar routes may reflect how much one is flexibly mapping (hippocampal place learning) or forming habitual responses to an environment (striatal response learning). To support this argument, we need to first link these putative systems to behaviors consistent with different navigational styles.

An elegant aspect of the dual-solution T-maze is that encoding is kept constant; place or response learning is manifested in behavior during retrieval. We present a novel dualsolution task in humans designed to conceptually parallel the T-maze to address how the preferential use of place and response mechanisms during learning might explain variability in human navigational performance by asking whether preferential engagement of the putative place and response systems in the brain during encoding could predict behavioral performance observed during retrieval. 


\section{LEARNING}
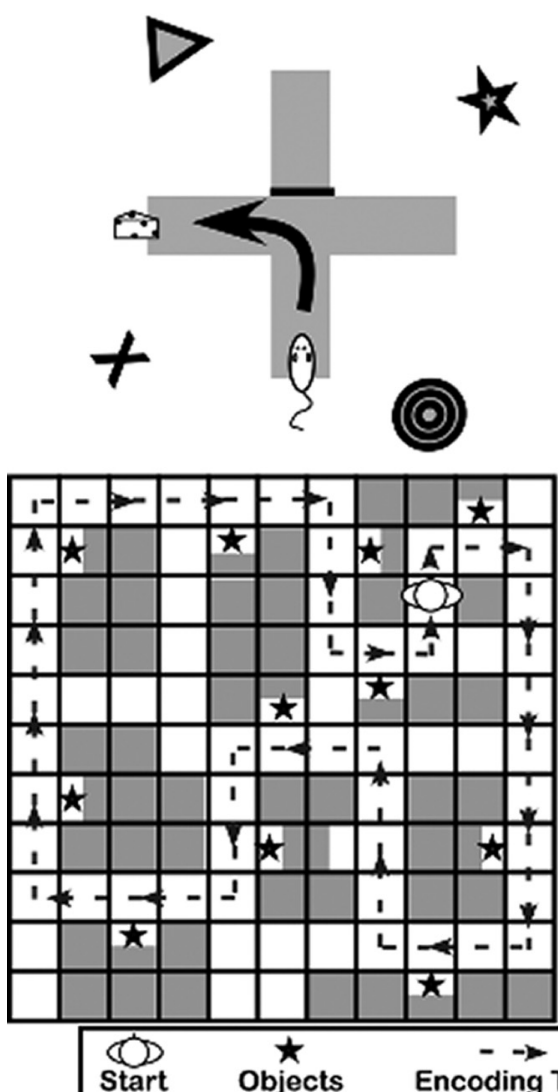

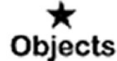

Encoding Tour

\section{TESTING}
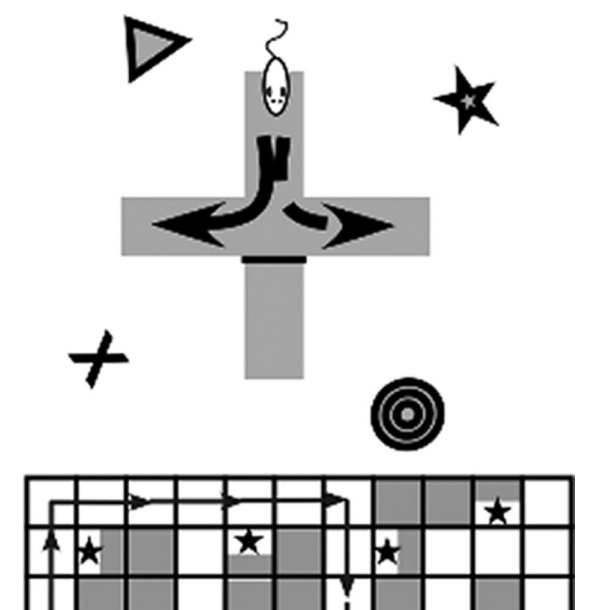

Figure 1. Dual-solution tasks designed for rats (top) and humans (bottom). In these paradigms, encoding is completed through repetition of the same set of responses in a stable environment. The critical test allows (at least) two solutions that reveal the type of strategy used.

\section{Materials and Methods}

Participants. One hundred twenty-eight adult participants (58 males, mean age $=19.72$ years, age range $18-24$ years) participated in the behavioral version of this experiment in return for extra credit in introductory psychology courses, and 20 adult participants $(10$ males, mean age $=$ 22.65 years, age range $=18-34$ years) participated in the fMRI experiment in return for financial compensation.

Materials and procedure. During the encoding phase, participants learned the locations of 12 objects in one of two $11 \times 11$ virtual environment (VE) mazes (constructed in Duke Nukem 3D; http// eduke32.com/) (Fig. 1, bottom) by watching repetitions of a $62 \mathrm{~s}$ video tour through the environment. The video tour always followed the same path through the environment and was repeated nine times.

Following the encoding phase, participants practiced navigating with arrow keys in an empty maze without choice points. Once participants were familiar with the controls, they completed 24 retrieval trials in which they were placed in the environment along the path observed during encoding and were given $45 \mathrm{~s}$ to navigate to one of the objects. To avoid any bias in strategy, participants began each trial facing a wall and were given a brief reorientation phase in which they were rotated a full $360^{\circ}$ over $\sim 6 \mathrm{~s}$.

Twelve trials were critical "shortcut-available" trials, where a novel shortcut offered a physically shorter path to the goal than the path experienced during encoding. The remaining trials were catch trials in which novel paths were equivalent to ("ambiguous," six trials) or physically longer than ("familiar fastest," six trials) the learned path.

Individual differences. Fifty-eight of the participants in the behavioral study also completed the Spatial Perspective Test (SPT) (Kozhevnikov and Hegarty, 2001) as a measure of ability to reason flexibly about spatial

information. In this test, participants view a simple display comprised of line drawings and then take a perspective within the display. Participants then draw a line to point to objects relative to this perspective. Participants complete 12 trials, and performance on this task is measured as a $180^{\circ}$ absolute average angular error.

Imaging procedures. In the fMRI version of the experiment, participants performed the dual-solution task but were scanned while encoding the VE. To adapt the task for the scanner, navigational control videos were interspersed with repetitions of the video tour during encoding. In this navigational control condition, participants saw videos of motion through random hallways in which they tracked red and blue spheres.

Participants completed three runs with three encoding and four control videos in each run. The dual-solution retrieval task immediately followed the scan session. To maximize power in the fMRI experiment, the proportion of shortcut trials was increased from 12 to 16 . Thirty-two of the 128 behavioral participants were run using this proportion of trial types to verify that the change did not produce a different distribution of place-response indices.

fMRI acquisition. Imaging sessions were conducted on a 3 Tesla Philips scanner equipped with a SENSE parallel imaging headcoil (MRI Devices) and higher-order shims to compensate for local field distortions located at the F.M. Kirby Research Center for Functional Brain Imaging at the Kennedy Krieger Institute in Baltimore, MD. Functional images were collected using a $\mathrm{T}^{\star}$-weighted echo-planar single-shot pulse sequence with an acquisition matrix of $72 \times 72$, an echo time of $35 \mathrm{~ms}$, a flip angle of $70^{\circ}$, a sense factor of 2 , an in-plane resolution of $3 \times 3 \mathrm{~mm}$, and a TR of $2 \mathrm{~s}$. Each volume consisted of 34 3-mm-thick axial slices with no gap, aligned parallel to the line from the anterior to the posterior commissures. Four dummy scans were completed at the beginning of each run to allow for stabilization of the MR signal. In addition, a structural whole-brain scan was acquired using an MP-RAGE T1-weighted sequence with 231 oblique slices, $0.65 \mathrm{~mm}$ isotropic resolution, and a field of view of $240 \mathrm{~mm}$.

Subject-level fMRI analysis. Image analysis was performed using the Analysis of Functional Neuroimages (AFNI) (Cox, 1996) software package. Functional images were coregistered and corrected for slice timing, and head motion was accounted for using a three-dimensional registration algorithm to remove trials in which a significant head motion occurred plus or minus one TR from further analysis. Functional runs were subsequently concatenated, and behavioral vectors were defined to model the encoding and control task conditions. These vectors were then used to model each individual's data using a deconvolution approach based on general linear regression using the control condition as a baseline. The resulting statistical fit coefficient maps represent the difference in activity between the encoding and control conditions for a given time point for a given voxel.

Cross-participant alignment. Methods used for cross-participant alignment in this study were previously described in detail (Kirwan and Stark, 2007; Yassa and Stark, 2009; Lacy et al., 2010). This method was developed to increase the power of multisubject regional fMRI studies by focusing alignment power to regions of interest using a manual segmentation of the subject's anatomical scan. First, affine registration was used to transform the subject's anatomical and functional images to the Talairach coordinate system (Talairach and Tournoux, 1988). The caudate, hippocampus, and the perirhinal, entorhinal, and parahippocampal cor- 
tices were manually segmented bilaterally using methods described by Insausti et al. (1998). Using the grayscale structural image for the pure cross-correlation error metric, Advanced Normalization Tools (Avants et al., 2008) was used to calculate the 3D vector field transformation for each subject needed to align the subject's structural data to a template based on the modal grayscale model based on the entire sample (Klein et al., 2009; Yushkevich et al., 2009). The resulting 3D vector field for each individual was then applied to the concatenated fit coefficient maps from the functional analysis.

Group analysis. Group data were modeled in AFNI using a hybrid functional/anatomical analysis. The primary contrast for all analyses was based on a two-tailed $t$ test contrasting the encoding and control conditions. To target our specific regions of interest, a liberal voxel threshold of $p<0.05$ was used on the overall $t$ statistic in combination with a spatial extent threshold of 50 contiguous voxels. The resulting areas of activation were then combined with the anatomical segmentation to only include voxels within the hippocampus and caudate. Voxels within each functional/anatomical region of interest were then used in the subsequent analyses.

\section{Results}

\section{Behavioral results}

The number of trials completed in each trial type and overall was calculated for each participant to verify successful navigation (average percentage of trials completed: $83 \%$ ). For the critical shortcut-available trials, we compared the number of steps made along one of the possible shortcut paths to the number of steps made along the familiar path using the algorithm of Dijkstra (1959). The trial was classified according to which path accounted for more of the navigational behavior. Occasionally, participants did not take either an optimal path or the familiar path, or failed to reach the goal location; these indeterminate trials did not contribute to the participant's behavioral index.

We calculated the proportion of trials on which participants took an available shortcut as an index of an individual's tendency toward place (reason flexibly) or response (stick to familiar) learning (place/response index). The frequency of different values on the place/response index revealed that people varied across the full range using $0-100 \%$ of the available shortcuts, with many people ranging in between (Fig. $2 a$ ). The correlation between the place/response index and the score on the SPT showed a strong positive relationship ( $r=0.37$, two-tailed $t$ test; $\left.t_{(56)}=2.98, p=0.004\right)$, indicating that greater flexibility in reasoning was associated with more use of shortcuts (Fig. $2 b$ ). Together, these results suggest that the place/response index is a dimension along which individuals show natural variability, and the connection between place performance and flexible spatial reasoning is consistent with a distinction between flexible place learning and rigid response learning (O'Keefe and Nadel, 1978; Packard and McGaugh, 1996; Squire, 2004; Foerde et al., 2006; Yin and Knowlton, 2006).

\section{fMRI results}

This smaller sample of participants completed an average of $89 \%$ of the trials and represented a place/response index ranging from 0.06 to 0.83 . As a manipulation check, we verified that our encoding task was consistent with the broader literature using a whole-brain analysis for VE encoding versus control with a more stringent height threshold $(p<0.001)$. As shown in Figure 3, we observed activation in the standard "navigation network" associated with environmental learning and memory (Maguire et al., 1998; Shelton and Gabrieli, 2002). With this validation of the paradigm, we then moved to the central question of how behavior relates to activation in the hippocampus and caudate.
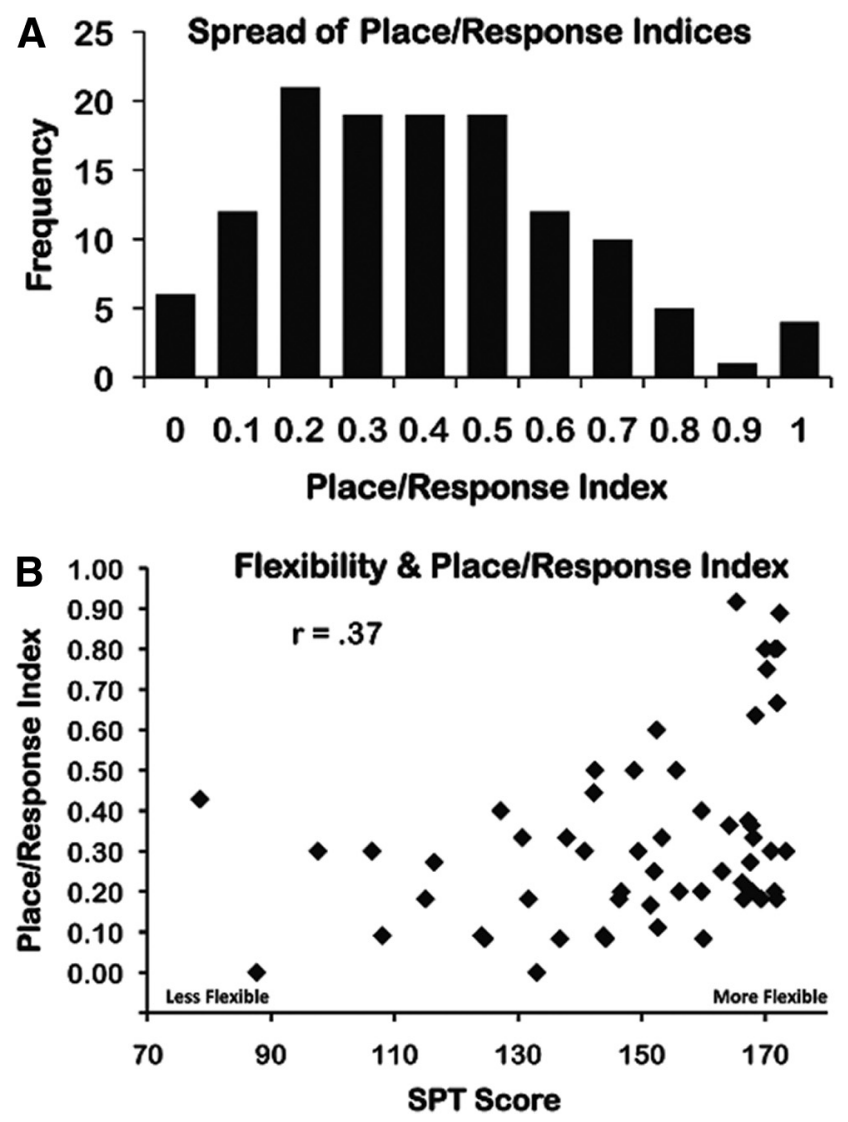

Figure 2. Behavioral results. $A$, The distribution of place/response indices for the behavioral sample, revealing full range. $\boldsymbol{B}$, Correlation between SPT performance, as a measure of spatial flexibility, and the place/response index ( $r=0.37, t_{(56)}=2.98, p=0.004$, two-tailed test). Participants who showed greater flexibility in spatial reasoning (i.e. higher SPT scores) took more available shortcuts than participants who showed less flexibility (i.e., lower SPT scores).

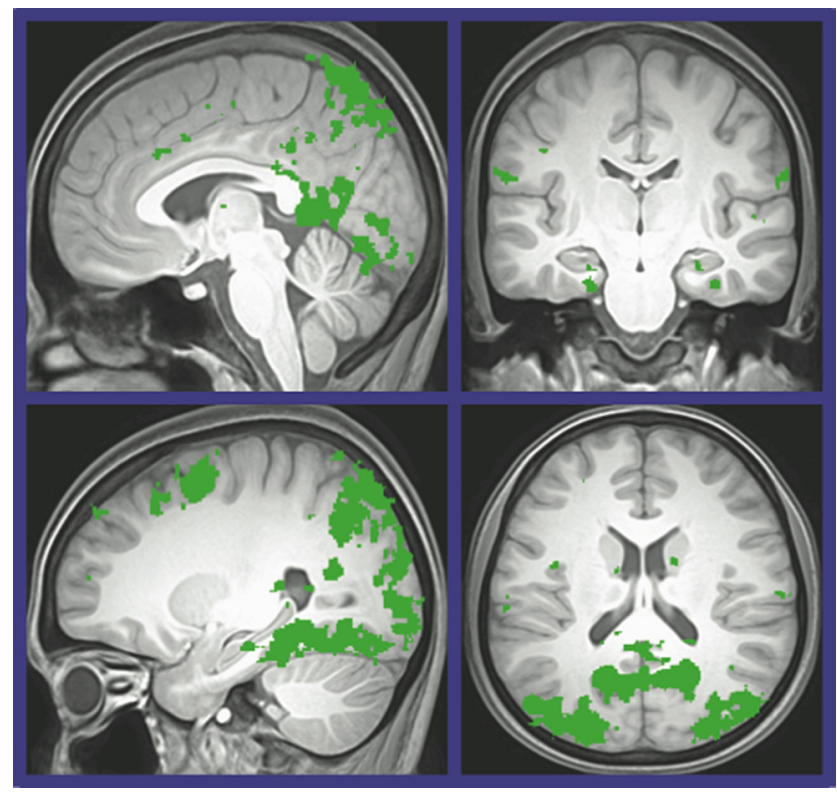

Figure 3. Regions identified in a whole-brain functional analysis for spatial encoding versus control.

To evaluate whether activation in the putative place and response regions predicted subsequent performance, we first identified regions of interest based on structure and function as described in the Materials and Methods section. We restricted 

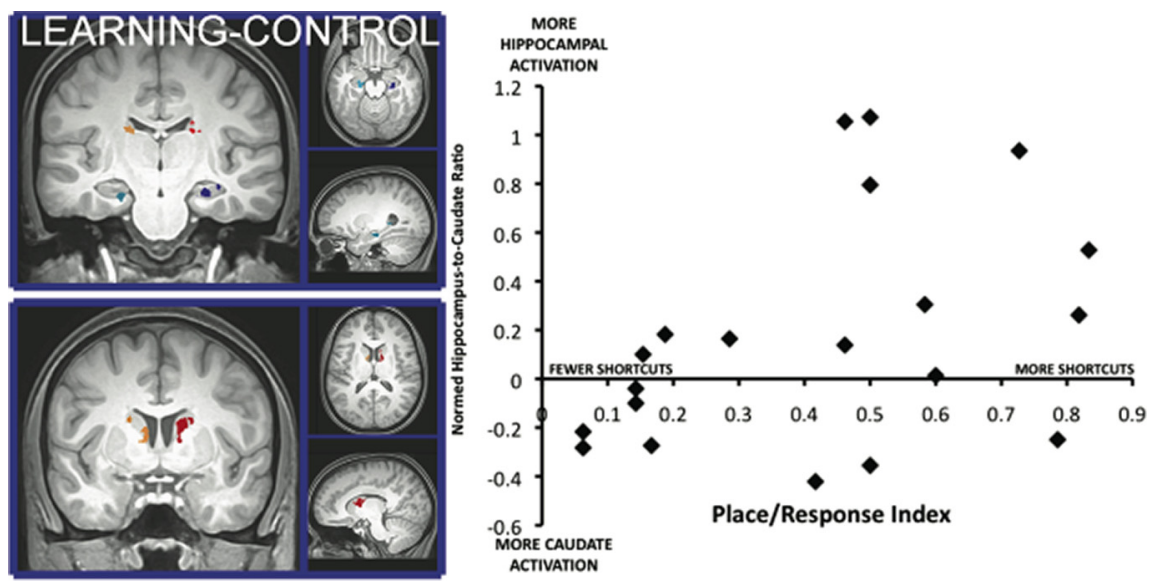

Figure 4. Region of interest analysis. Activation in the hippocampus and caudate identified via learning-control contrast and structural segmentation (left) was extracted and converted to a normed ratio [(hippocampus - caudate)/(hippocampus + caudate)] in order to correlate with the place/response index (right). Individuals who showed greater hippocampal activation used more shortcuts (more place-like).

our analysis to regions of interest based on the hypotheses from the rodent literature (bilateral hippocampus and caudate). However, these regions are embedded within broader learning and memory networks, and we emphasize that their functions must be interpreted in the context of the memory systems they exemplify, rather than as discrete modules of processing. In this light, we measured individual differences in the relative activation of these two regions.

Voxel selection for the regions of interest resulted in the following four areas of activation (Fig. 4, left): bilateral hippocampus (623 and 307 voxels in the right and left, respectively); and bilateral caudate nucleus (465 and 149 voxels in the right and left, respectively). Mean activity in these clusters was then used to calculate a normed ratio of hippocampal/striatal activation. For each participant, we calculated the place/response index at retrieval. We then correlated the normed ratio of hippocampal/striatal activation with the place/response index. As shown in Figure 4, this resulted in a striking correlation $(r=$ 0.44 , two-tailed $t$ test; $t_{(18)}=2.10, p=0.05$ ), with the activation ratio predicting $19 \%$ of the variance of subsequent memory performance.

To ensure that this relationship was not driven by the contribution of a single region, we correlated activation in each region separately with the place/response index. Although neither separate correlation reached statistical significance (place/response index and hippocampus: $r=0.31$, two-tailed $t$ test; $t_{(18)}=1.43$; $p=0.18$; place/response index and caudate: $r=-0.26$, twotailed $t$ test; $t_{(18)}=1.14 ; p=0.27$ ), they are each in the direction predicted by the place and response literature in rodents. These data reinforce our suggestion that the balance between memory systems during learning is more important than either one alone.

Finally, we verified that this relationship could not be attributed to differences in overall learning. We performed a median split on the participants based on their place/response index. Participants in the place range ( 14.3 goals found) and response range (13.4 goals found) did not differ in terms of their ability to find goals $\left(t_{(9)}=1.57, p=0.15\right)$. In addition, we observed no evidence of a correlation between the normed ratio of hippocampal/striatal activation and the number of critical trials completed $(r=$ -0.1 , two-tailed $t$ test; $\left.t_{(18)}=0.426, p=0.67\right)$. Together, the relative engagement of memory systems during encoding predicts navigational strategy rather than the success of navigation. As in the T-maze used in rodents, this suggests that how people choose to navigate may depend on the differential engagement of specific learning mechanisms during encoding.

\section{Discussion}

Previous studies have suggested that humans use place- and response-like mechanisms in different situations akin to those predicted from the animal models (Hartley et al., 2003; Iaria et al., 2003; Voermans et al., 2004; Iglói et al., 2010). We bring a new twist to this story by demonstrating that the preferential engagement of these putative mechanisms can be used to explain some of the variability in human navigation. More importantly, our results were not based on whether their navigation was a success or failure. Our participants successfully arrived at the target locations within the time constraints and with limited wandering, whereas they differed with respect to the paths chosen during navigation. As such, the activation of the place and response systems was not predicting whether people navigated successfully but rather how they navigated successfully. This provides a novel approach to navigational styles with broadreaching implications.

First, we suggest that preferential engagement of place and response learning may be a dimension along which people vary. An important observation was the continuous nature of both our measure of place and response and the relative engagement of hippocampus and caudate. That is, some people fell in the tails of the continuum, but many showed a mixture of solutions across trials. Similarly, participants tended to show activation in both the hippocampus and caudate, with variability in both regions. This is consistent with a model in which humans can avail themselves of multiple mechanisms during learning (for review, see Schacter and Tulving, 1994; Squire, 2004). Critically, we observed that the balance of the engagement of the underlying neural mechanisms during encoding could predict the balance of the use of the familiar path and the use of shortcuts at retrieval that could not be captured by a simple all-or-none classification. These data imply that when there are no specific constraints on the strategies that can or should be used, individuals can differ with respect to how they naturally weight the contributions of multiple memory systems. We suggest that this preferential weighting at learning reflects a tendency to encode spatial information more readily in one system or the other, as evidenced by what they choose to do in subsequent navigation.

To capture these findings, we suggest a potential model in which individuals naturally engage both place and response learning mechanisms but have a baseline preference/bias for how they will balance the engagement of those systems. This bias is reflected in the brain activation during encoding when the conditions of retrieval may or may not be evident. Thus, it appears that the balance of activation in these brain systems may reflect individual differences in approaches to spatial learning rather than explicit encoding of one solution or another. In other words, our participants were likely learning about both the structure of the environment and the repeated set of navigational responses but differed with respect to the degree of learning that occurred in each system. We argue that this may provide mechanistic support for the emergence of different strategies for solving the same navigational problem. For 
example, a bias toward place learning might support more global strategies that emphasize flexible understanding, whereas a bias toward response learning might support more path-based strategies that emphasize repetition of effective routes.

In the current study, navigational style was measured in the selection of particular strategies during retrieval when the conditions would allow either system to prevail. The correlation between this behavior and the brain activation highlights the fact that how people approached encoding had a clear impact on how they selected to approach retrieval. However, our model also makes predictions about what different people should be able to do under different navigational constraints rather than simply what they "choose" to do under unconstrained retrieval conditions. In addition to accounting for some aspects of individual differences (Lawton, 1994; Pazzaglia and De Beni, 2001; Fields and Shelton, 2006), this work provides a foundation for investigating how these two systems might be differentially used within and across individuals. Thinking in terms of not only one's initial biases but also how environmental conditions, individual skill sets, and navigational goals might cause people to use one strategy over another will enrich our overall understanding of how humans navigate.

Finally, this work represents a critical bridge between the systems neuroscience of rodent spatial learning mechanisms and real-world behavior in human navigation. This opens the door for taking questions about such aspects as neuromodulation, hormones, genetics, and aging that have been articulated in the rat and applying them to the complexities of human navigational behavior. Together, these investigations would revolutionize our approach by providing access to a wider range of factors to explain the enormous variability in human spatial learning and navigation.

\section{References}

Avants B, Duda JT, Kim J, Zhang H, Pluta J, Gee JC, Whyte J (2008) Multivariate analysis of structural and diffusion imaging in traumatic brain injury. Acad Radiol 15:1360-1375.

Cox RW (1996) AFNI software for analysis and visualization of functional magnetic resonance neuroimages. Comput Biomed Res 29:162-173.

Dijkstra EW (1959) A note on two problems in connexion with graphs. Numer Math 1:269-271.

Fields AW, Shelton AL (2006) Individual skill differences and large-scale environmental learning. J Exp Psychol Learn Mem Cogn 32:506-515.

Foerde K, Knowlton BJ, Poldrack RA (2006) Modulation of competing memory systems by distraction. Proc Natl Acad Sci U S A 103:1177811783.

Hartley T, Maguire EA, Spiers HJ, Burgess N (2003) The well-worn route and the path less traveled: distinct neural bases of route following and wayfinding in humans. Neuron 37:877-888.

Iaria G, Petrides M, Dagher A, Pike B, Bohbot VD (2003) Cognitive strategies dependent on the hippocampus and caudate nucleus in human navigation: variability and change with practice. J Neurosci 23:5945-5952.

Iglói K, Doeller CF, Berthoz A, Rondi-Reig L, Burgess N (2010) Lateralized human hippocampal activity predicts navigation based on sequence or place memory. Proc Natl Acad Sci U S A 107:14466-14471.

Insausti R, Juottonen K, Soininen H, Insausti AM, Partanen K, Vainio P, Laakso MP, Pitkänen A (1998) MR volumetric analysis of the human entorhinal, perirhinal, and temporopolar cortices. Am J Neuroradiol 19:659-671.

Kirwan CB, Stark CE (2007) Overcoming interference: an fMRI investigation of pattern separation in the medial temporal lobe. Learn Mem 14:625-633.
Klein A, Andersson J, Ardekani BA, Ashburner J, Avants B, Chiang MC, Christensen GE, Collins DL, Gee J, Hellier P, Song JH, Jenkinson M, Lepage C, Rueckert D, Thompson P, Vercauteren T, Woods RP, Mann JJ, Parsey RV (2009) Evaluation of 14 nonlinear deformation algorithms applied to human brain MRI registration. Neuroimage 46:786-802.

Korol DL, Gold PE (2007) Modulation of learning and memory by adrenal and ovarian hormones. In: Neurobiology of learning and memory (Kesner RP, ed), pp 243-268. New York: Elsevier.

Kozhevnikov M, Hegarty M (2001) A dissociation between object manipulation spatial ability and spatial orientation ability. Mem Cognit 29: 745-756.

Lacy JW, Yassa MA, Stark SM, Muftuler LT, Stark CE (2010) Distinct pattern separation related transfer functions in human CA3/dentate and CA1 revealed using high-resolution fMRI and variable mnemonic similarity. Learn Mem 18:15-18.

Lawton CA (1994) Gender differences in way-finding strategies: relationship to spatial ability and spatial anxiety. Sex Roles 30:765-779.

Maguire EA, Burgess N, Donnett JG, Frackowiak RS, Frith CD, O'Keefe J (1998) Knowing where and getting there: a human navigation network. Science 280:921-924.

Morris RGM (1981) Spatial localization does not require the presence of local cues. Learn Motiv 12:239-260.

O'Keefe J, Nadel L (1978) The hippocampus as a cognitive map. Oxford, UK: Clarendon.

Packard MG, McGaugh JL (1996) Inactivation of hippocampus or caudate nucleus with lidocaine differentially affects expression of place and response learning. Neurobiol Learn Mem 65:65-72.

Packard MG, Hirsh R, White NM (1989) Differential effects of fornix and caudate nucleus lesions on two radial maze tasks: evidence for multiple memory systems. J Neurosci 9:1465-1472.

Pazzaglia F, De Beni R (2001) Strategies of processing spatial information in survey and landmark-centered individuals. Eur J Cogn Psychol 13: 493-508.

Restle F (1957) Discrimination of cues in mazes: a resolution of the "placevs.-response" question. Psychol Rev 64:217-228.

Ritchie BF, Aeschlimm B, Pierce P (1950) Studies in spatial learning. VIII. Place performance and the acquisition of place dispositions. J Comp Physiol Psychol 43:73-85.

Sage JR, Knowlton BJ (2000) Effects of US devaluation on win-stay and win-shift radial maze performance in rats. Behav Neurosci 114:295-306.

Schacter DL, Tulving E (eds) (1994) Memory systems. Cambridge MA: MIT.

Shelton AL, Gabrieli JD (2002) Neural correlates of encoding space from route and survey perspectives. J Neurosci 22:2711-2717.

Squire LR (2004) Memory systems of the brain: a brief history and current perspective. Neurobiol Learn Mem 82:171-177.

Talairach J, Tournoux P (1988) A co-planar stereotaxic atlas of the human brain. New York: Thieme Medical.

Tolman EC (1948) Cognitive maps in rats and men. Psychol Rev 55: 189-208.

Tolman EC, Ritchie BF, Kalish D (1946) Studies in spatial learning. II. Place learning versus response learning. J Exp Psychol 36:221-229.

Voermans NC, Petersson KM, Daudey L, Weber B, Van Spaendonck KP, Kremer HP, Fernández G (2004) Interaction between the human hippocampus and the caudate nucleus during route recognition. Neuron 43:427-435.

Yassa MA, Stark CE (2009) A quantitative evaluation of cross-participant registration techniques for MRI studies of the medial temporal lobe. Neuroimage 44:319-327.

Yin HH, Knowlton BJ (2006) The role of the basal ganglia in habit formation. Nat Rev Neurosci 7:464-476.

Yushkevich PA, Avants BB, Pluta J, Das S, Minkoff D, Mechanic-Hamilton D, Glynn S, Pickup S, Liu W, Gee JC, Grossman M, Detre JA (2009) A high-resolution computational atlas of the human hippocampus from postmortem magnetic resonance imaging at 9.4 T. Neuroimage 44:385398. 